\title{
Who suffered most after deaths due to COVID-19? Prevalence and correlates of prolonged grief disorder in COVID-19 related bereaved adults
}

Suqin Tang ${ }^{1 *}$ (D) and Zhendong Xiang ${ }^{2}$

\begin{abstract}
Background: Deaths by COVID-19 have left behind nearly 12 million recent bereaved individuals worldwide and researchers have raised concerns that the circumstances of COVID-19 related deaths will lead to a rise prevalence of prolonged grief disorder (PGD) cases. However, to date, no studies have examined the prevalence of PGD among people bereaved due to COVID-19. This study aimed to estimate the prevalence of PGD and investigated demographic and loss-related factors associated with prolonged grief symptoms among Chinese individuals bereaved due to COVID-19.

Methods: This was a cross-sectional online survey conducted between September 1 and October 3, 2020. A total of 422 Chinese participants (55.5\% males; 32.73 [9.31] years old) who lost a close person due to COVID-19 participated in the study. Demographic and loss-related information was collected, and self-reported prolonged grief symptoms were measured by a 13-item International Prolonged Grief Disorder Scale (IPGDS: 1-65) and a 17item Traumatic Grief Inventory Self Report (TGI-SR: 1-85). Multiple linear regression analysis was used to determine the associated factors of levels of grief symptoms.

Results: Prevalence of PGD was 37.8\% screened by IPGDS and 29.3\% by TGI-SR. No difference was found in levels of grief symptoms between participants whose close one died more than 6 months ago and those who experienced the loss less than 6 months ago. More severe prolonged grief symptoms assessed by IPGDS was associated with losing a close person by COVID-19 rather than complications (B: 5.35; 95\% Cl: 0.54-10.05), losing a partner (B: 7.80; 95\% Cl: 3.24-12.37), child (B: 8.15; 95\% Cl: 1.03-15.26), and parent (B: 5.49; 95\% Cl: 1.49-9.48) rather than losing a relative or a person with other relationship, feeling more traumatic about the loss (B: 1.71; $95 \% \mathrm{Cl}$ : 0.52-2.90), being closer with the deceased (B: 1.60; 95\% Cl: 0.34-2.86). Moreover, Losing a grandparent (B: 6.62; 95\% Cl: $0.53-12.71)$ and having more conflicts with the deceased (B: $1.05 ; 95 \% \mathrm{Cl}:-0.008-2.11)$ were related to higher levels of grief symptoms assessed by TGI-SR.

(Continued on next page)
\end{abstract}

\footnotetext{
* Correspondence: jasminetsq@hotmail.com

'Department of Sociology, Law School, Shenzhen University, L3-1217, Zhili

Building, Canghai Campus, Shenzhen 618010, China

Full list of author information is available at the end of the article
}

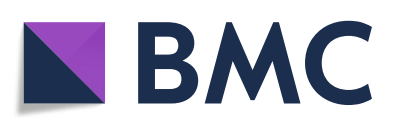

(- The Author(s). 2021 Open Access This article is licensed under a Creative Commons Attribution 4.0 International License, which permits use, sharing, adaptation, distribution and reproduction in any medium or format, as long as you give appropriate credit to the original author(s) and the source, provide a link to the Creative Commons licence, and indicate if changes were made. The images or other third party material in this article are included in the article's Creative Commons licence, unless indicated otherwise in a credit line to the material. If material is not included in the article's Creative Commons licence and your intended use is not permitted by statutory regulation or exceeds the permitted use, you will need to obtain permission directly from the copyright holder. To view a copy of this licence, visit http://creativecommons.org/licenses/by/4.0/ The Creative Commons Public Domain Dedication waiver (http://creativecommons.org/publicdomain/zero/1.0/) applies to the data made available in this article, unless otherwise stated in a credit line to the data. 
(Continued from previous page)

Conclusions: Echoing researchers' concerns, the prevalence of PGD is high among people bereaved due to COVID19. Individuals with a higher risk of developing PGD should be identified and bereavement support should be offered as early as possible.

Keywords: Bereavement, Prolonged grief disorder, Persistent complex bereavement disorder, Prevalence, COVID-19, China

\section{Background}

By January 8, 2021, there have been over 86 million confirmed cases of coronavirus disease (COVID-19), including more than 1.8 million deaths [1]. As shown in a recent study, for each COVID-19 death, about 9 family members would be affected and grieve [2]. It is estimated that the mass bereavement due to COVID-19 will leave behind nearly 16 million newly bereaved people globally. If deaths of close friends were counted, this number could be even larger. Given the lasting impacts on the bereaved population brought by the COVID-19 pandemic, delivering efficient care for people who bereaved due to COVID-19 becomes a worldwide challenge.

After a close person died, 9.8\% of people would develop prolonged grief disorder (PGD) [3], a newly added diagnosis in the International Classification of Diseases eleventh edition (ICD-11) [4]. PGD is a persistent and pervasive grief response characterized by longing for the deceased and/or persistent preoccupation with the deceased, accompanied by intense emotional pain including sadness, guilt, anger, denial, blame, difficulty accepting the death, feeling one has lost a part of one's self, an ability to experience positive mood and so on. This grief response has persisted for an atypically long period of time following the loss (more than 6 months at a minimum) and clearly exceeds expected social, cultural, or religious norms for the individual's culture and context, and results in significant impairment in personal, family, occupational and other important areas of functioning. The Diagnostical and Statistical Manual of Mental Disorders fifth edition (DSM-5) published in 2013 included Persistent Complex Bereavement Disorder in Section 3 under Conditions for Further Study [5] and is considered to be replaced by a diagnosis also named prolonged grief disorder in Section 2 under Diagnostic Criteria and Codes in the forthcoming version of the DSM-5 [6, 7].

Researchers have raised concerns that the circumstances of COVID-19 related deaths will lead to a worldwide elevated prevalence of PGD cases [8, 9]. First, COVID-19 related deaths are usually unexpected, and the unexpectedness of death yielded higher levels of prolonged grief symptoms in Australian [10], Dutch [11], Japanese [12], Chinese [13], and Thai [14] bereaved individuals. Second, COVID-19 related deaths could be traumatic as people may experience the pandemic as a disaster. Survivors of unnatural deaths like disasters and accidents are at a higher level of developing prolonged grief $[15,16]$. The traumatic nature of COVID-19 related deaths is also manifested by being unable to visit the dying person and hold and attend traditional funerals and other rituals. A study revealed that among bereaved whose family members died in ICU, the likelihood of developing prolonged grief for those who did not have the chance to say goodbye to the deceased person was 2 to 3 times to other bereaved individuals [17]. As the subjective experience of traumatic death increases, the severity of prolonged grief symptoms increases [18].

Despite the consensus that mental health professionals should pay attention to the rise of prolonged grief disorder after the COVID-19 pandemic $[8,9]$ and call for evidence-based and culturally sensitive bereavement care for individuals bereaved during the pandemic [19-24], no study specifically focused on bereaved people and the impact that a death linked to a pandemic had on their subsequent grief before the outbreak of the COVID-19 pandemic [25]. After the COVID-19 outbreak, one empirical study included 49 Dutch individuals who experienced COVID-19 related bereavement showed that they reported more severe grief than people who experienced natural losses and equivalent levels of grief with people who experienced unnatural losses [26]. Other than that, little is known about the prevalence and symptom severity of prolonged grief disorder among people whose close ones died from the COVID-19 pandemic.

In order to identify at-risk bereaved people who experienced deaths due to COVID-19, a number of lossrelated factors and their association with the prolonged grief symptom severity should be examined. First, previous studies have demonstrated that closer kinship was related to higher levels of prolonged grief symptoms across age groups, causes of death, and cultures [13, 16, 27-35]. In addition to the objective nature of the relationship between the deceased and the bereaved, the quality of their relationship matters. Quality of mournerdecedent relationship contains two salient factors, namely closeness and conflict with the deceased [36]. According to the attachment-based model, losing a close person represents the loss of an important attachment figure, and the more deeply one was attached to the 
deceased person, the more difficult it for him/her to integrate the reality of death and update the mental representation of the deceased [37]. On the other hand, clinical observations showed that conflicts exist at the time of death and earlier may lead to pathological mourning [38]. Hence, it is worthwhile to investigate the role of the closeness and conflict with the deceased in relation to prolonged grief. Last, as mentioned earlier, unexpected death [10-14] and losses with traumatic features [15-18] tended to yield more severe prolonged grief symptoms.

Therefore, this study aimed to examine the prevalence and severity of prolonged grief symptoms and to investigate demographic and loss-related factors associated with prolonged grief symptoms among individuals bereaved due to COVID-19. We hypothesized that a) the prevalence and severity of prolonged grief would be high, b) closer kinship with the deceased would be related to more severe symptoms, c) higher levels of unexpectedness and traumatic experience of the death/loss would be correlated with higher grief symptom levels, and d) both closeness and conflict with the deceased would be related to grief symptoms levels.

\section{Methods}

\section{Participants and procedure}

Data collection was conducted from September 1, 2020, to October 3, 2020. To be eligible to participate in the study, participants had to be 18 and/or above years old and lose a close one due to COVID-19. Participants were recruited through social network websites (e.g., Baidu, WeiBo) and mobile applications (e.g., WeChat). A brief introduction of the study and inclusion criteria for participants were stated, and a link to the online survey was posted in the recruiting advertisement. Once the participants accessed the online survey via the link, the consent page including the purpose of the study, voluntariness of participation, confidentiality, and grief support resources would be presented before entering the formal survey. Only when the participants ticked the box "I understand the information described above and agree to participate in this study", they would enter the formal survey. At the end of the survey, support resources specifically for bereaved people during the COVID-19 were listed, including free counseling and grief counseling information, self-help resources, and books. Ethical approval was obtained before data collection from the Ethics Committee of the Health Science Center, Shenzhen University.

A sample of 476 participants submitted the questionnaire, and 54 of whom were excluded from the analysis due to response time less than $5 \mathrm{~min}(n=21)$, inconsistent information about the deceased person $(n=15)$, bereaved more than 9 months ago $(n=11)$, and patterned responses $(n=8)$. Therefore, data of 422 participants were analyzed.

\section{Measures \\ Demographic and loss-related information}

Demographics included sex, age, education, and religious belief. Loss-related information included relationship to deceased (i.e., partner, child, parent, grandparent, relative, friend, other relationship), age of deceased, times since loss in months, and cause of death (i.e., COVID-19 and COVID-19 related complication). Moreover, unexpectedness of death, traumatic level of loss, closeness with deceased, and conflict with deceased were measured by single items with a 5 -point Likert scale from 1 (not at all) to 5 (very much).

\section{International ICD-11 prolonged grief disorder scale (IPGDS)}

\section{[39]}

The IPGDS was developed for assessing symptoms of PGD in ICD-11. The standard scale of the IPGDS contains 13 items about the yearning, preoccupation, emotional distress, and functioning impairment after the death of a close person, and one cultural screening item. Participants indicated how often they experienced these symptoms in the past month on a 5-point Likert scale from 1 (almost never) to 5 (always). A total score of all items excluding the cultural screening item represents the symptom levels of PGD, with higher scores indicating higher levels of symptom. PGD strict criteria were adopted to estimate the prevalence rates: one or two of items 1 or 2,1 or more of items $3-12$, and the impairment criterion (item 13) all rated 4 or above [40]. The IPGDS was validated in both Chinese and Germanspeaking samples, and the 13 core IPGDS items revealed a two-factor structure that accounted for $57.9 \%$ of the variance and received reliability of 0.93 in the Chinese sample [39]. In the current sample, the 13-item IPGDS also resulted in a two-factor structure that explained $53.3 \%$ of the variance, and the Cronbach's alpha was 0.89 .

\section{Traumatic grief inventory self report (TGI-SR) [41, 42]}

The full version of TGI-SR was administered, and the 17 -item version of TGI-SR was used for data analysis as it was developed to assess symptoms of PCBD in DSM5 , including yearning, preoccupation, reactive distress to the death, social/identity disruption, and functioning impairment. Participants indicated how often they experienced these symptoms in the past month on a 5-point Likert scale from 1 (never) to 5 (always). A total score of all 17 items represents the symptom levels of PCBD, with higher scores indicating more severe symptoms. The provisional PCBD diagnosis can be made by treating each item rated as 4 or 5 as a symptom endorsed and 
then follow the DSM-5 based diagnostic rule, which requires endorsement of (a) at least 1 Criterion B item, (b) at least 6 Criterion $\mathrm{C}$ items, and (c) the Criterion D item $[41,42]$. Since items of the TGI-SR were from the Inventory of Complicated Grief (ICG) [43], the Inventory Complicated Grief-Revised (ICG-R) [44], and the DSM-5 descriptions of PCBD symptoms [5], and the two inventories and the DSM-5 manual were validated in the Chinese samples $[45,46]$, the content validity of the Chinese version of the TGI-SR should be sufficient. Similar to its original version $[41,42]$, the scree plots indicated that one-factor solutions accounting for $40.05 \%$ of the variance adequately represented the data of the current sample, and factor loadings of all items were larger than 0.40. The Cronbach's alpha of the 17-item TGISR was 0.95 in the bereaved patient sample [41], 0.90 in the disaster-bereaved sample [42], and 0.91 in the current sample.

\section{Statistical analyses}

The characteristics of the sample were summarized using descriptive statistics. Cronbach's alpha coefficients measured the internal reliability of the IPGDS and the TGI-SR; a cut-off of .70 was used to indicate good internal reliability. Cohen's kappa was calculated to assess the consistency of screening positive cases by IPGDS and TGI-SR. Multiple linear regression analyses were performed to examine the independent associations between demographic and loss-related characteristics and grief symptoms. The associations were reported as or unstandardized coefficient $B$, standardized coefficient beta, and $95 \%$ confidence intervals (CIs). $P$ values $<0.05$ were considered statistically significant (two-sided). Analyses were performed using IBM SPSS Statistics 26.

\section{Results}

\section{Sample characteristics}

Table 1 summarized the demographic and loss-related characteristics of the sample. Participants were $32.73 \pm$ 9.31 years old, ranging from 18 to 73 . Men accounted for $55.5 \%$ of the sample. Most of the participants had received higher education (79.4\%) and no religious belief (93.6\%). Participants who had lost their partner consisted of $32.9 \%$ of the sample, followed by those who lost a parent (23.0\%), a grandparent (16.4\%), and a friend (15.2\%). Participants experienced the death $5.10 \pm 1.72$ months ago. In general, participants were unexpected for the death, perceived the loss as traumatic, and close to the deceased.

\section{Prevalence and symptom levels of PGD and PCBD}

The mean score of PGD symptoms was $41.58 \pm 9.60$ and varied between 18 and 60 . The average score of PCBD symptoms was $54.07 \pm 12.28$ and ranged from 22 to 78 .
Table 1 Demographic and loss-related information ( $N=422)$

\begin{tabular}{lll}
\hline Variable & M / & SD / $\%$ \\
\hline Age & 32.73 & 9.31 \\
Sex & & \\
$\quad$ Male & 234 & $55.5 \%$ \\
$\quad$ Female & 188 & $44.5 \%$ \\
Education & & \\
$\quad$ Junior secondary school and/or below & 21 & $5.0 \%$ \\
$\quad$ Senior secondary school & 66 & $15.6 \%$ \\
$\quad$ College & 320 & $75.8 \%$ \\
$\quad$ Postgraduate and/or above & 15 & $3.6 \%$ \\
Religious belief & & \\
$\quad$ No & 395 & $93.6 \%$ \\
$\quad$ Yes &
\end{tabular}

Role of deceased

$\begin{array}{lll}\text { Partner } & 139 & 32.9 \%\end{array}$

$\begin{array}{lll}\text { Child } & 24 & 5.7 \%\end{array}$

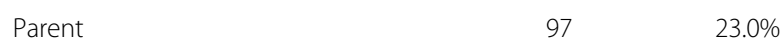

Grandparent $\quad 69 \quad 16.4 \%$

$\begin{array}{lll}\text { Relative }^{\mathrm{b}} & 22 & 5.2 \%\end{array}$

Friend $\quad 64 \quad 15.2 \%$

$\begin{array}{lll}\text { Other }^{c} & 7 & 1.7 \%\end{array}$

Age of deceased $\quad 47.81 \quad 21.55$

Time since loss in months $\quad 5.10 \quad 1.72$

Cause of death

COVID-19 $408 \quad 96.7 \%$

COVID-19 related complication $^{d} \quad 14 \quad 3.3 \%$

$\begin{array}{lll}\text { Unexpectedness of death } & 3.67 & 1.23\end{array}$

$\begin{array}{lll}\text { Traumatic level of loss } & 3.86 & 0.97\end{array}$

$\begin{array}{lll}\text { Closeness with deceased } & 4.15 & 0.88\end{array}$

Conflict with deceased $\quad 1.76 \quad 1.11$

$\begin{array}{lll}\text { PGD symptom levels } & 41.58 & 9.60\end{array}$

$<6$ months post-loss ${ }^{\mathrm{e}} \quad \mathbf{4 1 . 3 7} \quad 9.61$

$\geq 6$ months post-loss ${ }^{\mathrm{f}} \quad 41.85 \quad 9.61$

$\begin{array}{lll}\text { PCBD symptom levels } & 54.07 & 12.28\end{array}$

$<6$ months post-loss ${ }^{\mathrm{e}} \quad 53.64 \quad 12.36$

$\geq 6$ months post-loss ${ }^{\mathrm{f}} \quad 54.61 \quad 12.19$

Note. $M$ Mean, $S D$ Standard Deviation, $P G D$ Prolonged Grief Disorder, $P C B D$ Persistent Complex Bereavement Disorder

a Religious belief included Buddhism $(n=17)$, Taoism $(n=3)$, Catholicism ( $n=$ 3), Christianism ( $(n=3)$, and Islamism $(n=1)$

${ }^{\mathrm{b}}$ Relative included uncle $(n=5)$, aunt $(n=4)$, cousin $(n=4)$, grandaunt $(n=3)$, granduncle $(n=1)$, great grandmother $(n=1)$, and not specified $(n=4)$

c Other relationship included colleagues $(n=4)$, acquaintance $(n=2)$, and not specified $(n=1)$

${ }^{d}$ COVID-19 related complication included heart disease $(n=2)$, fever $(n=2)$, acute respiratory distress syndrome $(n=1)$, asthma $(n=1)$, cardiovascular and cerebrovascular diseases $(n=1)$, chronic obstructive pulmonary disease $(n=1)$, diabetes $(n=1)$, high blood pressure $(n=1)$, liver cancer $(n=1)$, lung cancer $(n=1)$, obesity $(n=1)$, and respiratory failure $(n=1)$

e $n=234$

${ }^{\mathrm{f}} n=188$ 
Notably, the grief symptom levels did not differ between participants who bereaved 6 months ago and the rest on either PGD symptoms [t $(420)=0.51, p=0.61]$ or PCBD symptoms [ $\mathrm{t}(420)=0.81, p=0.42]$. Means and standard deviations of PGD and PCBD symptoms are presented in Table 1.

Participants whose close ones died 6 months ago $(n=188)$ formed a subsample to approximate the prevalence rates of PGD and PCBD. Seventy-one (37.8\%) of this subsample met the PGD criteria and 55 (29.3\%) met the PCBD criteria. There were 18 participants who met the PGD criteria but not the PCBD criteria, and two participants met the PCBD criteria but not the PGD criteria, kappa $=0.76 \pm 0.05, p<$ 0.001 , indicating the two diagnostic algorithms were significantly consistent. Furthermore, 69 (36.7\%) participants indicated that "my grief would be considered worse (e.g., more intense, severe and/or of longer duration) than for others from my community or culture", yet only half of them met the PGD criteria $(n=38)$ and the PCBD criteria $(n=33)$.

\section{Correlates of PGD and PCBD symptoms}

To investigate which factor correlates with PGD and PCBD symptom the most, multiple linear regression analyses were conducted, with all demographic and lossrelated variables were entered as independent variables. The model for PGD was significant, F $(18,403)=6.72$, $p<0.001$, and explained $23.1 \%$ of the variance. The model for PCBD was also significant, $F(18,403)=7.02$, $p<0.001$, and explained $23.9 \%$ of the variance. Both regression models identified that COVID-19 rather than its complications as the cause of death, losing a partner, child, and parent, traumatic levels of the loss, and closeness with the deceased as associated factors of grief symptoms (see Table 2). Moreover, losing a grandparent and conflicts with the deceased were related to PCBD symptoms.

\section{Discussion}

This is the first study to estimate the prevalence of PGD in ICD-11 and PCBD in DSM-5 and to identify demographic and loss-related factors associated with PGD and PCBD symptoms in a large sample of individuals bereaved due to COVID-19. Using the latest diagnostic criteria of pathological grief in ICD-11 and DSM-5 and the most updated measures for PGD and PCBD, this study

Table 2 Regression analyses of PGD and PCBD

\begin{tabular}{|c|c|c|c|c|c|c|c|c|}
\hline & PGD & & & & PCBD & & & \\
\hline Variable & B (SE) & $95 \% \mathrm{Cl}$ & $\beta$ & $t$ & $\mathrm{~B}(\mathrm{SE})$ & $95 \% \mathrm{Cl}$ & $\beta$ & $t$ \\
\hline Age & $-0.10(0.07)$ & $-0.25,0.04$ & -0.10 & -1.40 & $-0.15(0.09)$ & $-0.33,0.04$ & -0.11 & -1.55 \\
\hline Sex & $0.52(0.89)$ & $-1.23,2.27$ & 0.03 & 0.58 & $0.99(1.13)$ & $-1.24,3.22$ & 0.04 & 0.87 \\
\hline \multicolumn{9}{|l|}{ Education ${ }^{a}$} \\
\hline Senior high school & $0.88(2.19)$ & $-3.43,5.19$ & 0.03 & 0.40 & $-0.11(2.79)$ & $-5.59,5.37$ & -0.003 & -0.39 \\
\hline College & $-1.72(2.05)$ & $-5.75,2.30$ & -0.08 & -0.84 & $-2.68(2.61)$ & $-7.80,2.45$ & -0.09 & -1.03 \\
\hline Postgraduate & $-1.69(2.99)$ & $-7.57,4.19$ & -0.03 & -0.57 & $-2.32(3.81)$ & $-9.80,5.16$ & -0.04 & -0.61 \\
\hline Religious belief ${ }^{b}$ & $2.15(1.87)$ & $-1.53,5.83$ & 0.06 & 1.15 & $2.78(2.38)$ & $-1.90,7.47$ & 0.06 & 1.17 \\
\hline Age of deceased & $-0.003(0.05)$ & $-0.11,0.10$ & -0.01 & -0.06 & $-0.01(0.07)$ & $-0.15,0.12$ & -0.02 & -1.17 \\
\hline Time since loss in months & $0.05(0.25)$ & $-0.45,0.55$ & 0.01 & 0.18 & $0.11(0.32)$ & $-0.52,0.75$ & 0.02 & 0.35 \\
\hline Cause of death ${ }^{c}$ & $5.35(2.45)$ & $0.54,10.05$ & 0.10 & $2.19^{*}$ & $7.77(3.12)$ & $1.64,13.89$ & 0.11 & $2.49^{*}$ \\
\hline \multicolumn{9}{|l|}{ Role of deceased ${ }^{d}$} \\
\hline Partner & $7.80(2.32)$ & $3.24,12.37$ & 0.38 & $3.36^{* *}$ & $10.73(2.95)$ & $4.92,16.54$ & 0.41 & $3.63^{* * *}$ \\
\hline Child & $8.15(3.62)$ & $1.03,15.26$ & 0.20 & $2.25^{*}$ & $10.27(4.61)$ & $1.21,19.32$ & 0.19 & $2.23^{*}$ \\
\hline Parent & $5.49(2.03)$ & $1.49,9.48$ & 0.24 & $2.70^{* *}$ & $8.39(2.59)$ & $3.30,13.47$ & 0.29 & $3.24^{* *}$ \\
\hline Grandparent & $4.46(2.43)$ & $-0.33,9.24$ & 0.17 & 1.83 & $6.62(2.59)$ & $0.53,12.71$ & 0.20 & $2.14^{*}$ \\
\hline Friend & $3.08(2.34)$ & $-1.52,7.68$ & 0.12 & 1.32 & $4.84(2.98)$ & $-1.01,10.69$ & 0.14 & 1.63 \\
\hline Unexpectedness of death & $-0.07(0.39)$ & $-0.83,0.70$ & -0.009 & -0.17 & $-0.09(0.49)$ & $-1.06,0.88$ & -0.009 & -0.19 \\
\hline Traumatic level of loss & $1.71(0.61)$ & $0.52,2.90$ & 0.17 & $2.82^{* *}$ & $2.23(0.77)$ & $0.71,3.74$ & 0.18 & $2.89^{* *}$ \\
\hline Closeness with deceased & $1.60(0.64)$ & $0.34,2.86$ & 0.14 & $2.50^{*}$ & $1.72(0.81)$ & $0.12,3.32$ & 0.12 & $2.11^{*}$ \\
\hline Conflict with deceased & $0.57(0.42)$ & $-0.26,1.40$ & 0.07 & 1.34 & $1.05(0.54)$ & $-0.008,2.11$ & 0.10 & $1.95+$ \\
\hline
\end{tabular}

Note. PGD Prolonged Grief Disorder, PCBD Persistent Complex Bereavement Disorder, SE Standard Error. CI Confidence Interval

${ }^{a}$ reference group: junior high school and below

b reference group: no religious belief

c reference group: COVID-19 related complication

$\mathrm{d}$ reference group: a combined group of relative and other relationships

$\dagger p=0.05,{ }^{*} p<0.05,{ }^{* *} p<0.01,{ }^{* * *} p<0.001$ 
demonstrates that over one-third of COVID-19 related bereaved individuals suffered from PGD or PCBD. Factors associated with more severe grief symptoms were losing a close person by COVID-19 rather than related complications, losing a partner, child, parent, grandparent, feeling more traumatic about the loss, being closer to the deceased, and having more conflicts with the deceased.

Prevalence of PGD found in our COVID-19 related bereaved sample $(37.8 \%)$ is higher than Chinese people bereaved 55 months ago (12.7\%) and German-speaking people bereaved 48 months ago (7.3\%); both studies used the same measure and diagnostic algorithm [39]. The number is also higher than the prevalence of PGD in a sample of bereaved Wenchuan earthquake survivors in China (8.47\%) [16] and a group of Chinese Shidu parents (i.e., parents who lost their only child) bereaved 7 years ago (22.2\%) [47], who are undergoing the most painful and traumatic experience than other types of loss and facing physical and psychological challenges [48]. The number is similar to the prevalence in a large-scale survey of Chinese Shidu parents bereaved 9 years ago (35.5\%) [49]. Except for the prevalence, the symptom levels of PGD $(41.85 \pm 9.61)$ were higher than those reported in the German-speaking sample $(29.22 \pm 10.83)$ and the Chinese sample $(36.29 \pm 11.35)$.

Rate of PCBD in our study (29.3\%) is higher than that in two Dutch bereaved patient samples, namely $17.7 \%$ [41] and $12.5 \%$ [42]. The number is also higher than that in Dutch citizens bereaved 344 days ago due to the Ukrainian plane disaster (6.6\%) [42]. While the prevalence of PCBD in our sample is higher than in previous studies, the symptom levels measured by the 18 -item version of TGI-SR $(57.39 \pm 13.10)$ seems equivalent to that reported among 49 Dutch people bereaved about 2 months ago due to COVID-19 (57.37 \pm 9.60) [26].

Not surprisingly, time since loss may help to explain the high prevalence of PGD and PCBD in the COVID19 bereaved population as the survey was conducted approximately 9 months since the first COVID-19 case reported in China, which was less than all of the previous studies. Nevertheless, while the time frame of the survey could be partially accounted for the relatively high prevalence of PGD and PCBD in the COVID-19 bereaved population, our findings still support concerns from researchers that there would be a rise in PGD $[8$, 9] because acute grief is among the strongest predictors of the development of PGD [50]. The finding that no difference of grief symptoms was detected between participants bereaved 6 months ago or less than 6 months echoes in part that acute grief might greatly correlate with prolonged grief, as the time criterion for PGD in ICD-11 was at least 6 months [4].
These findings add to the discussion on the PGD and PCBD criteria in terms of the symptom criteria and the time criterion. The first question is that whether the symptom criteria should be stricter for unnatural death as grief reactions for unnatural death might be more severe in nature, as shown in this study and another study on deaths due to the COVID-19 pandemic [26]. Second, as severe grief reactions last for more than 6 months in the context of the pandemic, which may imply that the duration of acute grief for mass bereavement could be longer, the time criterion in ICD-11 for diagnosing PGD (i.e., at least 6 months) remains questionable. Therefore, some scholars are advocating for extending the time criterion to 12 months [51]. On the other hand, however, it is possible that under the circumstance of mass bereavement, a shortened time criterion would facilitate providing timely help for those in need.

Another interesting point is that over one-third of participants who bereaved for more than 6 months believed their grief reactions went beyond cultural norms, yet only half of them met the symptom and functioning criteria. This reflects a discrepancy in the understanding of "abnormal grief" between the bereaved and professionals: in the case of bereavement experience due to COVID-19, some bereaved individuals tended to believe that their grief went wrong even though their grief was assessed to be within the normal range in terms of both severity and functioning impairment from the professionals' perspective. Efforts should be made to narrow the gap of understanding of pathological grief and to seek consensus between the bereaved and the professionals regarding the consideration for social, cultural, or religious norms [52].

Although the diagnostic algorithms of PGD and PCBD in this study were generally consistent, prevlance of PCBD (29.3\%) was lower than that of PGD (37.8\%), suggesting that the DSM-5 PCBD criteria may be more strict. A previous study compared six proposed diagnostic criteria sets for disturbed grief and also found lower prevalence of DSM-5 PCBD (11.1\%) than ICD-11 PGD (19.8\%) [7]. The inconsistency between the two diagnoses was believed to bring negative impact on research and care [53], and debate on which diagnosis is more clinical useful is ongoing. Some found that hightening the threshold of ICD-11 PGD criteria could increase its agreement with DSM-5 PCBD [53], whereas the others showed that ICD-11 PGD symptoms were bettercaptured psychopathology, more sensitive to change over time [54], and more responsive to treatment [55].

Most of the associated factors identified in this study are consistent with previous research. First, verifying our hypothesis, those who grieve the most were the ones who lost a first-degree family member (i.e., partner, parent, child), followed by grandparents (a significant 
correlate for PCBD symptoms), friends, relatives, and other relationships. This pattern strengthens the importance of considering the kinship between the bereaved and the deceased across causes of death [13, 16, 27-34]. Second, although all participants lost their close ones due to COVID-19, a handful of them endorsed COVID19 induced complications as the causes of death and they experienced less severe grief symptoms than their peers. Despite the fact that deaths during the pandemic were generally unexpected, since those who died from complications was older [59.14 \pm 16.79 versus $47.42 \pm$ $21.61, \mathrm{t}(420)=2.008, p=0.045]$ and usually had a history of diseases, the bereaved might be more mentally prepared for the death than others, and preparedness for death served as a protective factor for grief symptoms $[35,56]$.

We found that in the grieving process during the pandemic, subjective traumatic level of the loss was more crucial than unexpectedness of the death in association to grief symptoms, which partially supports our hypotheses. Compared to the objective traumatic deaths (e.g., violent deaths), subjective experience of death as traumatic was a more significant factor that contributing to grief symptoms [18]. During the COVID-19 pandemic, because of the nationwide lockdown measures taken to prevent the spread of the virus in China, people were unable to celebrate the Spring Festival by gathering together in late January, hold funerals and farewell rituals for the deceased, offer condolences to bereaved families by physical company, and memorialize decedents by sweeping the tomb on the Tsing Ming Festival in April. Additionally, owning to the contagious nature of the pandemic, family members were not allowed to say goodbye to the dying patient, let alone keeping some meaningful personal belongings of the deceased [57]. All these created obstacles to the grieving process, which may result in prolonged grief.

Confirming our hypothesis, quality of relationship between the bereaved and the deceased also plays a part concerning grief severity and our findings suggest that assessing the ambivalence in the relationship is necessary as both closeness and conflict with the deceased were positively correlated with grief severity. The closer to the deceased before the death, the stronger attachment was established with the deceased. The bond of attachment would continue even when they are separated by death, yet it might imply disbelief that the person is dead and thus lead to unresolved grief [58]. Death of an attachment figure presents a "temporarily irreconcilable mismatch between an unrevised mental representation of a loved one and a dramatic change in the ongoing relationship with that person" (p.454), resulting in acute grief symptoms such as yearning for the deceased, preoccupation of the deceased, and loss of interest in the world [59]. Once the mental representation of the deceased was revised by incorporating the reality of death, acute grief symptoms would be resolved [37]. However, for individuals who are deeply attached to the deceased, the revision of the mental representation could take a longer time, and thus manifested as prolonged grief symptoms. Another finding we would like to highlight is that more conflicts with the deceased before the death, although at a smaller magnitude than closeness, is related to more severe PCBD symptom. It is the first study, to our knowledge, to provide empirical evidence for the relationship between conflict with the deceased and mental health. Conflicts existed at the time of death and earlier are assumed to be accompanied by a strong psychological dependence on the deceased [38], and greater dependency is a risk factor for prolonged grief symptoms [60].

This study contributes to the clinical practice for bereaved people in several aspects. First, as our findings confirmed the concerns about the rise of PGD after the outbreak of COVID-19 and the pandemic tends to be sustained, mental health practitioners need to be alert when caring for the bereaved and assess whether and when their grief goes pathological. Second, when assessing acute and prolonged grief of bereaved people, practitioners need to be aware of the difference between the ICD-11 and DSM-5 diagnostic guidelines for pathological grief and both guidelines should be considered in order to avoid omitting those in need. Third, not only those who had lost their first-degree relatives, but also those who had lost their grandparents and friends need to be included when providing bereavement care. Fourth, it is important to understand how the COVID19 pandemic had changed the bereaved people's perception of the death and their experience of the grieving process, as the subjective traumatic levels of the loss served a significant correlates to elevated grief symptoms. Last, quality of the relationship between the deceased and the bereaved is worth exploring, and both positive and negative memories should be examined to resolve the unfinished business and thus to facilitate a more adaptive grieving process.

This study also has limitations. First, the sample is recruited by convenience sampling, whose representativeness may be affected by the self-selection bias. The current sample might experience less severe grief symptoms than a random sample, as it may be more taxing for those who suffered from more severe grief symptoms to fill out all questions. Thus, the study may underestimate the severity of PGD and PCBD symptoms among COVID-19 bereaved individuals. Second, although strictly following the ICD-11 and DSM-5 diagnostic guidelines, this study adopted self-reported measures rather than structured clinical interviews to determine the 
prevalence rates. Similarly, data on whether the death was due to COVID-19 was reported by the participants rather than extracted medical records. Third, due to the cross-sectional design, effects of demographic and lossrelated variables in predicting the development of PGD and PCBD could not be examined. Longitudinal study is needed to determine the causality. Last, loss-related characteristics specifically affected by the COVID-19 pandemic was not included in explaining the variance of grief symptoms. Future research can investigate the relationship between grieving experience altered by COVID19 and the development of PGD and PCBD so that bereavement support could be tuned for the COVID-19or pandemic-related bereaved population.

Notwithstanding these considerations, the study contributes to the field by providing the first evidence of the prevalence, symptom severity, and associated factors of PGD and PCBD in a sample of COVID-19 bereaved individuals. It compared the prevalence of pathological grief between ICD-11 and DSM-5 in a Chinese population, adding cross-cultural data of adopting these two diagnostic systems in the midst of utilizing and revising the diagnostic criteria $[7,61]$.

\section{Conclusions}

The prevalence of PGD in COVID-19 bereaved people was $37.8 \%$ and that of PCBD was $39.9 \%$. Factors associated with more severe PGD and PCBD symptoms were losing a close person by COVID-19 rather than complications, losing a partner, child, parent, grandparent, feeling more traumatic about the loss, being closer to the deceased, and having more conflicts with the deceased. The high prevalence found in this study validates concerns and early interventions for people bereaved due to COVID-19, and a targeted group that requires more support could be identified based on the associated factors reported in this study.

\section{Acknowledgements}

We thank all participants who took part in this study and two undergraduate students from Department of Sociology, Law School, Shenzhen University, Qianxin Chen and Yi Yu, for their help in monitoring the data collection process and setting up the preliminary dataset.

\section{Authors' contributions}

ST designed the study, interpreted the data, and wrote and revise the manuscript; ZX analyzed the data. All authors reviewed and approved the final version of the manuscript.

\section{Funding}

This work was supported by the Guangdong Planning Office of Philosophy and Social Science [grant number GD20YSH06] and the Shenzhen University Natural Science Research Grant [grant number 860-000002110172].

\section{Availability of data and materials}

The datasets used and/or analysed during the current study are available from the corresponding author on reasonable request.

\section{Ethics approval and consent to participate}

This study was approved by the Ethics Committee of the Health Science Center, Shenzhen University. All participants and their parents were informed of the objectives of the study and completing the survey implied consent to participate in the study.

\section{Consent for publication}

Not applicable.

\section{Competing interests}

The authors declare that they have no competing interests.

\section{Author details}

'Department of Sociology, Law School, Shenzhen University, L3-1217, Zhili Building, Canghai Campus, Shenzhen 618010, China. ${ }^{2}$ Shenzhen Yishi Huolala Technology Limited Company, Futian District, Shenzhen 518049, China.

Received: 23 November 2020 Accepted: 4 February 2021

Published online: 11 February 2021

\section{References}

1. World Health Organization: WHO coronavirus disease (COVID-19) dashboard 2021; [cited 2021 Jan 9] Available from: https://covid19.who.int/

2. Verdery AM, Smith-Greenaway E, Margolis R, et al. Tracking the reach of COVID-19 kin loss with a bereavement multiplier applied to the United States. PNAS. 2020;117:17695-701.

3. Lundorff $M$, Holmgren $H$, Zachariae $R$, et al. Prevalence of prolonged grief disorder in adult bereavement: a systematic review and meta-analysis. J Affect Disord. 2017;212:138-49.

4. World Health Organization: International classification of diseases (11th ed.) 2018; [cited 2018 Jun 9] Available from: https://icd.who.int/dev11/l-m/en\#/ http\%3a\%2f\%2fid.who.int\%2ficd\%2fentity\%2f1183832314

5. American Psychiatric Association. Diagnostic and statistical manual of mental disorders. 5th ed. Arlington, VA: American Psychiatric Publishing; 2013.

6. American Psychiatric Association: Addition of a new diagnosis, "prolonged grief disorder," to the depressive disorders chapter 2020; [cited 2020 Oct 6] Available from: https://www.psychiatry.org/psychiatrists/practice/dsm/ proposed-changes

7. Boelen PA, Lenferink LIM. Comparison of six proposed diagnostic criteria sets for disturbed grief. Psychiatry Res. 2020;285:112786.

8. Eisma MC, Boelen PA, Lenferink LIM. Prolonged grief disorder following the coronavirus (COVID-19) pandemic. Psychiatry Res. 2020;288:113031.

9. Johns L, Blackburn P, McAuliffe D. COVID-19, prolonged grief disorder and the role of social work. Int Soc Work. 2020;63:660-4.

10. Ingles J, Spinks C, Yeates $L$, et al. Posttraumatic stress and prolonged grief after the sudden cardiac death of a young relative. JAMA Intern Med. 2016; 176:402-5.

11. Boelen PA, van Denderen $M$, de Keijser J. Prolonged grief, posttraumatic stress, anger, and revenge phenomena following homicidal loss: the role of negative cognitions and avoidance behaviors. Homicide Stud. 2016;20:17795.

12. Fujisawa D, Miyashita M, Nakajima S, et al. Prevalence and determinants of complicated grief in general population. J Affect Disord. 2010;127:352-8.

13. He L, Tang S, Yu W, et al. The prevalence, comorbidity and risks of prolonged grief disorder among bereaved Chinese adults. Psychiatry Res. 2014;219:347-52.

14. Wanna R, Lueboonthavatchai P. Prevalance of complicated grief and associated factors in psychiatric outptients at King Chulalongkorn Memorial Hospital. J Psychiatric Assoc Thailand. 2015;60:85-98.

15. Djelantik AAAMJ, Smid GE, Mroz A, et al. The prevalence of prolonged grief disorder in bereaved individuals following unnatural losses: systematic review and meta regression analysis. J Affect Disord. 2020;265:146-56.

16. Yi X, Gao J, Wu C, et al. Prevalence and risk factors of prolonged grief disorder among bereaved survivors seven years after the Wenchuan earthquake in China: a cross-sectional study. Int J Nurs Sci. 2018:5:157-61.

17. Kentish-Barnes N, Chaize M, Seegers V, et al. Complicated grief after death of a relative in the intensive care unit. Eur Respir J. 2015:45:1341-52.

18. Tang S, Chow AYM. Subjective and objective traumatic death: distinct roles in developing complicated grief and depression among older adults in Hong Kong. Int Psychogeriatr. 2017;29:409-17. 
19. Harrop E, Mann M, Semedo L, et al. What elements of a systems' approach to bereavement are most effective in times of mass bereavement? A narrative systematic review with lessons for COVID-19. Palliat Med. 2020;34: 1165-81.

20. Kokou-Kpolou CK. Prolonged grief related to COVID-19 deaths: do we have to fear a steep rise in traumatic and disenfranchised griefs? Psychol Trauma Theory Res Pract Policy. 2020;12:S94-5.

21. Morris SE, Moment A. Thomas J deLima: caring for bereaved family members during the COVID-19 pandemic: before and after the death of a patient. J Pain Symptom Manag. 2020;60:e70-4.

22. Selman LE, Chao D, Sowden R, et al. Bereavement support on the rrontline of COVID-19: recommendations for hospital clinicians. J Pain Symptom Manag. 2020;60:e81-6.

23. Carr D, Boerner K, Moorman S. Bereavement in the time of coronavirus: unprecedented challenges demand novel interventions. J Aging Soc Policy. 2020;32:425-31.

24. Lichtenthal WG, Roberts KE, Prigerson HG. Bereavement care in the wake of COVID-19: offering condolences and referrals. Ann Int Med. 2020; [cited 2020 Oct 5] Available from: https://www.acpjournals.org/doi/10.7326/M20-2 526.

25. Mayland CR, Harding AJE, Preston N, et al. Supporting adults bereaved through COVID-19: a rapid review of the impact of previous pandemics on grief and bereavement. J Pain Symptom Manag. 2020;60:e33-9.

26. Eisma MC, Tamminga A, Smid GE, et al. Acute grief after deaths due to COVID-19, natural causes and unnatural causes: an empirical comparison. J Affect Disord. 2021:278:54-6.

27. Hibberd R, Elwood LS, Galovski TE. Risk and protective factors for posttraumatic stress disorder, prolonged grief, and depression in survivors of the violent death of a loved one. J Loss Trauma. 2010;15:426-47.

28. Mizuno Y, Kishimoto J, Asukai N. A nationwide random sampling survey of potential complicated grief in Japan. Death Studies. 2012;36:447-61.

29. Newson RS, Boelen PA, Hek K, et al. The prevalence and characteristics of complicated grief in older adults. J Affect Disord. 2011;132:231-8.

30. Stammel N, Heeke C, Bockers E, et al. Prolonged grief disorder three decades post loss in survivors of the Khmer rouge regime in Cambodia. J Affect Disord. 2013;144:87-93.

31. Li J, Chow AYM, Shi Z, et al. Prevalence and risk factors of complicated grief among Sichuan earthquake survivors. J Affect Disord. 2015;175:218-23.

32. van Denderen $\mathrm{M}$, de Keijser J, Huisman M, et al. Prevalence and correlates of self-rated posttraumatic stress disorder and complicated grief in a community-based sample of homicidally bereaved individuals. J Int Violence. 2016;31:207-27.

33. Lobb EA, Kristjanson LJ, Aoun SM, et al. Predictors of complicated grief: a systematic review of empirical studies. Death Stud. 2010;34:673-98.

34. Tang S, He L, Wang J, et al. Prevalence and risk factors of prolonged grief disorder among Chinese bereaved adults: evidence from mainland China and Hong Kong. Int J Psychol. 2016;51:91.

35. Tsai W-I, Prigerson HG, Li C-Y, et al. Longitudinal changes and predictors of prolonged grief for bereaved family caregivers over the first 2 years after the terminally ill cancer patient's death. Palliat Med. 2015; 0269216315603261.

36. Bottomley JS, Smigelsky MA, Floyd RG, et al. Closeness and conflict with the deceased: exploring the factor structure of the quality of relationships inventory in a bereaved student sample. Omega (Westport). 2019;79:377-93.

37. Shear KM, Shair H. Attachment, loss, and complicated grief. Dev Psychobiol. 2005;47:253-67.

38. Krupp G, Genovese F, Krupp T. To have and have not: multiple identifications in pathological bereavement. J Am Acad Psychoanal. 1986;14: 337-48.

39. Killikelly C, Zhou N, Merzhvynska M, et al. Development of the International Prolonged Grief Disorder Scale for the ICD-11: measurement of core symptoms and culture items adapted for Chinese and German-speaking samples. J Affect Disord. 2020; [cited 2020 Sep 23] Available from: http:// www.sciencedirect.com/science/article/pii/S016503272032663X.

40. Lenferink LIM, Boelen PA, Smid GE, et al. The importance of harmonising diagnostic criteria sets for pathological grief. Br J Psychiatry J Ment Sci. 2019:1-4.

41. Boelen PA, Smid GE. The traumatic grief inventory self-report version (TGISR): introduction and preliminary psychometric evaluation. J Loss Trauma. 2017:22:196-212.
42. Boelen PA, Djelantik AAAMJ, de Keijser J, et al. Further validation of the traumatic grief inventory-self report (TGI-SR): a measure of persistent complex bereavement disorder and prolonged grief disorder. Death Studies. 2019:43:351-64

43. Prigerson HG, Maciejewski PK, Reynolds lii CF, et al. Inventory of complicated grief: a scale to measure maladaptive symptoms of loss. Psychiatry Res. 1995;59:65-79.

44. Prigerson HG, Jacobs SC: Traumatic grief as a distinct disorder: A rationale, consensus criteria, and a preliminary empirical test, In Stroebe MS, Hansson $\mathrm{RO}$, Stroebe W, et al., editors Handbook of bereavement research: Consequences, coping, and care. Washington, DC, American Psychological Association, 2001, pp 613-647.

45. He L, Wang J, Tang S, et al. Reliability and validity of the inventory of complicated grief-revised. Chin Ment Health J. 2013;27:937-43.

46. Li J, Prigerson HG. Assessment and associated features of prolonged grief disorder among Chinese bereaved individuals. Compr Psychiatry. 2016;66:916.

47. Zhang $H$, Shang Z, Wu L, et al. Prolonged grief disorder in Chinese Shidu parents who have lost their only child. Eur J Psychotraumatol. 2020;11: 1726071.

48. Yin Q, Shang Z, Zhou N, et al. An investigation of physical and mental health consequences among Chinese parents who lost their only child. BMC Psychiatry. 2018;18:45

49. Zhou N, Wen J, Stelzer E-M, et al. Prevalence and associated factors of prolonged grief disorder in Chinese parents bereaved by losing their only child. Psychiatry Res. 2020;284:112766.

50. Boelen PA, Lenferink LIM. Symptoms of prolonged grief, posttraumatic stress, and depression in recently bereaved people: symptom profiles, predictive value, and cognitive behavioural correlates. Soc Psychiatry Psychiatr Epidemiol. 2020;55:765-77.

51. Prigerson $\mathrm{HG}$, Boelen PA, Xu J, et al. Validation of the new DSM-5-TR criteria for prolonged grief disorder and the PG-13-revised (PG-13-R) scale. World Psychiatry. 2021;20:96-106.

52. Killikelly C, Maercker A. Prolonged grief disorder for ICD-11: the primacy of clinical utility and international applicability. Eur J Psychotraumatol. 2017:8 [cited 2018 Nov 7] Available from: https://www.ncbi.nlm.nih.gov/pmc/a rticles/PMC5990943/.

53. Boelen PA, Lenferink LIM, Smid GE. Further evaluation of the factor structure, prevalence, and concurrent validity of DSM-5 criteria for persistent complex bereavement disorder and ICD-11 criteria for prolonged grief disorder. Psychiatry Res. 2019;273:206-10.

54. Bonanno GA, Malgaroli M. Trajectories of grief: comparing symptoms from the DSM-5 and ICD-11 diagnoses. Depress Anxiety. 2020;37:17-25.

55. Mauro C, Reynolds CF, Maercker A, et al. Prolonged grief disorder: clinical utility of ICD-11 diagnostic guidelines. Psychol Med. 2018:1-7.

56. Nielsen MK, Neergaard MA, Jensen AB, et al. Predictors of complicated grief and depression in bereaved caregivers: a nationwide prospective cohort study. J Pain Symptom Manag. 2017:53:540-50.

57. $\mathrm{Xu} \mathrm{J,} \mathrm{He} \mathrm{L,} \mathrm{Wang} \mathrm{J.} \mathrm{The} \mathrm{bereaved} \mathrm{one's} \mathrm{grief} \mathrm{reaction} \mathrm{characteristics} \mathrm{and}$ suggestions for grief counseling under the COVID-19 epidemic. Chin Ment Health J. 2020;34:382-6.

58. Field NP. Unresolved grief and continuing bonds: an attachment perspective. Death Stud. 2006;30:739-56.

59. Shear KM, Monk T, Houck P, et al. An attachment-based model of complicated grief including the role of avoidance. Eur Arch Psychiatry Clin Neurosci. 2007;257:453-61.

60. Thomas K, Hudson P, Trauer T, et al. Risk factors for developing prolonged grief during bereavement in family carers of cancer patients in palliative care: a longitudinal study. J Pain Symptom Manag. 2014;47:531-41.

61. Cozza SJ, Shear KM, Reynolds CF, et al. Optimizing the clinical utility of four proposed criteria for a persistent and impairing grief disorder by emphasizing core, rather than associated symptoms. Psychol Med. 2020;50: $438-45$

\section{Publisher's Note}

Springer Nature remains neutral with regard to jurisdictional claims in published maps and institutional affiliations. 